\title{
Gastroduodenal injury after radioembolization of hepatic tumors
}

Cristina Carretero, M.D., ${ }^{1}$ Miguel Munoz-Navas, M.D., Ph.D., ${ }^{1}$ Maite Betes, M.D., Ph.D., ${ }^{1}$ Ramon Angos, M.D., Ph.D., ${ }^{1}$ Jose C. Subtil, M.D., Ph.D., ${ }^{1}$ Ignacio FernandezUrien, M.D., ${ }^{1}$ Susana De la Riva, M.D., Ph.D., ${ }^{1}$ Josu Sola, M.D., Ph.D., ${ }^{2}$ Jose I. Bilbao, M.D., Ph.D., ${ }^{3}$ Esther de Luis, M.D., ${ }^{3}$ and Bruno Sangro, M.D., Ph.D. ${ }^{4}$

${ }^{1}$ Department of Gastroenterology, ${ }^{2}$ Department of Pathology, ${ }^{3}$ Department of Radiology, and ${ }^{4}$ Liver Unit, University Clinic of Navarra, Navarra, Spain

\section{BACKGROUND}

Radioembolization is a new tool for the treatment of hepatic tumors that consists in the injection of biocompatible microspheres carrying radioisotopes into the hepatic artery or its branches.

\section{METHODS}

We have performed radioembolization in 78 patients with hepatic tumors using resinbased microspheres loaded with yttrium-90. All patients were previously evaluated to minimize the risk of hazardous irradiation to nontarget organs and to obtain the data needed for dose calculation.

\section{RESULTS}

We report a complication found in three cases (3.8\%) that consists of abdominal pain resulting from gastroduodenal lesions and that had a chronic, insidious course. Microscopically, microspheres were detected in the specimens obtained from all affected gastric areas. Since these gastroduodenal lesions do not appear when nonradiating microspheres are injected in animals, lesions are likely to be due to radiation and not to an ischemic effect of vascular occlusion by spheres.

\section{CONCLUSIONS}

We believe that a pretreatment evaluation that includes a more thorough scrutiny of the hepatic vascularization in search of small collaterals connecting to the gastroduodenal tract can help prevent this awkward complication.

Reprint requests and correspondence: Cristina Carretero.

Department of Gastroenterology, Clinica Universitaria de Navarra.

Pio XII, 36, 31008 Pamplona, Navarra, Spain. 


\section{INTRODUCTION}

Radioembolization is a new tool for the treatment of hepatic tumors. It consists of the delivery of resin or glass microspheres loaded with radioactive isotopes into the hepatic artery or its branches. SIR-Spheres ${ }^{\circledR}$ (Sirtex Medical Europe, Bonn, Germany) are resin microspheres of around 35 microns in diameter loaded with yttrium-90, a beta-emitter with a halflife of about $64 \mathrm{~h}$ and an average penetration in tissues of around $2.4 \mathrm{~mm}$. Microspheres are embolized into the hepatic artery and they become lodged within the microvasculature of the tumor where they emit the beta radiation (1). SIR-Spheres allow, on average, 200-300 Gy to be delivered to liver tumors irrespective of their number, size, and location (2). We have retrospectively reviewed all symptomatic gastroduodenal lesions appearing after liver radioembolization in the largest population of patients so far studied.

\section{RADIOEMBOLIZATION PROCEDURE}

All patients give signed informed consent before pretreatment evaluation, which is designed to minimize the risk of hazardous irradiation to nontarget organs and to obtain the data needed for dose calculation. Evaluation starts with a comprehensive angiographic study aimed at detecting any possible variation of arterial liver distribution, identifying vessels that give arterial blood supply to every liver tumor nodule and those other vessels that may give access to extrahepatic organs, and evaluating portal vein blood flow. Then, the gastroduodenal artery (GDA) and any other collateral vessel that may result in microspheres being lodged into the gastrointestinal area are occluded by standard angiographic methods (normally endovascular coils). The GDA was occluded at evaluation in every case with two exceptions: those patients that already had the GDA occluded due to previous intra-arterial chemotherapy and those patients in whom the microspheres were injected from either the right hepatic artery or the left hepatic artery (lobar treatments) with the tip of the catheter placed far enough from the origin of the GDA. And finally, with the tip of the catheter placed in the position intended for treatment, $\mathrm{Tc}^{99}$-radiolabeled macroaggregates of albumin (MAA) are injected and subsequently imaged to determine the degree of shunt to the lung, to detect any possible "nontarget locations" of microspheres in the GI tract, and to evaluate the relative amount of activity going to the liver tumors and the nontumoral liver. One to two weeks later and with the tip of the catheter placed in the same position used for MAA test, the calculated activity of microspheres is injected. Following treatment, patients remain in hospital overnight and supportive therapy generally consists of (a) pre- and post-therapy IV hydration, (b) prophylaxis of gastritis with a proton pump inhibitor starting the day of treatment and continued for $4 \mathrm{wk}$, (c) a low-dose of methylprednisolone given for 4 wk starting on the day of treatment, and (d) antiemetics (e.g., ondansetron) and analgesics (e.g., paracetamol or tramadol) on demand.

\section{PATIENTS}

From September 2003 to June 2006, we have performed 80 radioembolization procedures in 78 patients with primary and secondary liver tumors. Three of them (3.8\%) presented significant abdominal pain 8-16 wk after the infusion of Sir-Spheres and underwent an upper endoscopy during the follow-up. 


\section{Case 1}

A 56-yr-old woman presented in 2003 having a papillary renal cell carcinoma with liver and pulmonary metastasis. Immunotherapy with interleukin 2 was started after nephrectomy but failed to induce tumor response. One year later, radioembolization was performed. Angiography and MAA test showed no contraindications for radioembolization, with an extrahepatic shunt of 5.85\%. A small gastric artery was occluded before microsphere injection at the same procedure and $1.11 \mathrm{GBq}$ of SIRSpheres were injected into the common hepatic artery. Twelve hours after the injection, the patient complained of right upper-abdominal pain, nausea, and vomiting. She received analgesia and prokinetics and the symptoms progressively improved but pain did not subside. Four months later, mild upper-abdominal pain still remained.

A FDG-PET-CT scan done for evaluation of tumor response showed a hypermetabolic area located in the pylorus (Fig. 1) suggestive of an inflammatory process. An upper endoscopy was then performed that revealed two ulcers in the duodenal bulb (Fig. 2). Urease test was negative and biopsies showed that the structure of all tissue layers was fairly well preserved, and that dark spheres were present in the submucosa (Fig. 3). Therapy with esomeprazole (40 mg daily) and pentoxifylline (thrice daily) resulted in symptom improvement and was maintained for 1 yr. On follow-up gastroscopy (3 months after the first one), a cicatricial area, probably related to previous ulcers, was apparent in the duodenal bulb, and close to it, a fibrin-coated ulcer of approximately 1 cm in diameter (Fig. 4).

\section{Case 2}

A 54-yr-old man presented in 2005 with small bowel neuroendocrine tumor and liver metastasis. Resection of the primary tumor was performed in 2002 and he subsequently received 4 courses of intra-arterial oxaliplatin and oral capecitabine. In May 2005, he was evaluated for radioembolization. No contraindications were found on angiography and MAA test, with an extrahepatic shunt of 5.23\%. The GDA was occluded before microsphere injection at the same procedure and $2.76 \mathrm{GBq}$ of SIR-Spheres were subsequently injected into the common hepatic artery. Intense pain developed during the procedure that required narcotics. Two months later, he was admitted because of abdominal pain, nausea, vomiting, and weight loss, and an upper endoscopy showed a wide ulcerated area involving the gastric antrum, lesser curvature, pylorus, and duodenal bulb (Fig. 5). Scattered fibrin-covered ulcers could be individualized within this area. Biopsy showed small spheres located inside the vessels (Fig. 6). He was placed on pantoprazole ( $40 \mathrm{mg}$ daily), and 10 months later he still had abdominal discomfort to some extent.

\section{Case 3}

A 48-yr-old man with nonresectable liver metastasis from colorectal cancer resected in 2004 had been treated with hepatic intra-arterial chemotherapy from February 2005 to March 2005 with no hepatic response. In May 2005, he was evaluated for radioembolization. No contraindications were found in the angiogram and MAA test with an extrahepatic shunt of $10.1 \%$. The GDA was occluded before microsphere injection at the same procedure and $1.11 \mathrm{GBq}$ of SIR-Spheres were injected into the common hepatic artery. Three months later during a routine evaluation, he complained of abdominal pain, nausea, and vomiting that had started 20 days after radioembolization. An upper endoscopy showed a large, welldelimited ulcer spreading from the posterior wall of the gastric body to the pylorus (Fig. 7) that was actively 
bleeding in the antrum. Treatment with esomeprazole (40 mg twice daily) and pentoxifylline (thrice daily) was followed by symptom improvement. A month later, a follow-up endoscopy showed that the surface of the ulcer was irregular with a big denudated area on which small islets of normal mucosa could be found, as if the mucosa was trying to regenerate (Fig. 8).

\section{DISCUSSION}

External irradiation of the stomach can cause severe gastritis and ulceration, which can lead to fatal bleeding and perforation (3). Most of the information about the tolerance dose of the stomach to radiation comes from clinical studies of radiotherapy in the treatment of Hodgkin's disease and testicular tumors (4), when the stomach is irradiated incidentally together with the upper abdominal lymph nodes. These studies have shown that a larger fraction size and a higher total dose are related to a higher rate of late radiation complications $(4,5)$. And in a rat model of radiation-induced gastric injury, animals that received between 25 and 28.5 Gy showed erosive and ulcerative gastritis (6). The spectrum of nonacute manifestations of radiation-induced GI lesions include dyspepsia, atrophic gastritis, and ulceration (7).

We have found a 3.8\% (3/78) incidence of symptomatic gastroduodenal ulcerations among patients receiving radioembolization for the treatment of liver tumors. This figure compares favorably with the only experience published so far (13\%, meaning 4 out of 30 patients) (2). The difference is likely to be due to a more comprehensive angiographic search of small collateral vessels connecting the hepatic artery with the GI tract with the help of a procedure that we have routinely performed lately. Planar images obtained after MAA injection, which is all that is needed to calculate lung shunt, were replaced by SPECT. And SPECT scan was then fused with the corresponding abdominal image (either CT or MRI) to improve the localization of activity both in the liver and in surrounding areas. This technique allows gastric uptake of MAA to be more clearly identified (Fig. 9). And since the routine implementation of this technique, we have not had any other gastroduodenal complication. Furthermore, in our opinion, it is absolutely mandatory to occlude the GDA in every case when treatment is performed in a whole-liver fashion from the proper or the common hepatic artery (8). Moreover, close attention has to be paid the day of the treatment to small collaterals arising from the common hepatic artery that may appear only after occlusion of the GDA.

Yet, we have retrospectively obtained and reviewed such fusion images from the cases here presented, and no significant activity was detected in the gastroduodenal area. The early onset of pain in some of our patients raises the possibility that plugged spheres might reduce blood flow due to occlusion of the vessels. Infrequently, there could also be a vessel spasm attributable to the catheter stimulus. Small collaterals might also develop during the time between evaluation and injection. After embolization of the GDA, there can be hypertrophy of the pyloric artery, which might result in unnoticed reflux of microspheres during the procedure.

From a mechanistic perspective, gastroduodenal lesions after radioembolization can result from ischemia, radiation, or both. The extent and homogeneity of large ulcers argues in favor of an ischemic effect since once would expect sphere deposition to be more heterogeneous. But gastroduodenal ulcers are not a common complication after 
chemoembolization for the treatment of hepatocellular carcinoma even when collateral occlusion is not performed earlier (9). We have studied the distribution and effects of "cold" microspheres (not containing Y-90) after massive injection into the hepatic artery of pigs without occluding collaterals. As shown in Figure 10, microspheres are located in the submucosa but no microscopic injury to the gastric or duodenal mucosa was observed. Although no animal received "hot" microspheres to be compared, this observation very strongly suggests that it is radiation and not ischemia that causes gastroduodenal ulcers after radioembolization.

In summary, hepatic radioembolization carries a risk of inducing actinic gastroduodenal ulcers due to misplacement of radioactive microspheres in the gastrointestinal tract. This risk is probably lower than $5 \%$ when a very active search for collateral vessels connecting the hepatic arteries to the GI area is performed. But abdominal pain persisting or developing 1-2 months after radioembolization should prompt the performance of an upper endoscopy that may rule out gastric lesions.

\section{STUDY HIGHLIGHTS}

What Is Current Knowledge

- $\quad$ Radioembolization can cause gastroduodenal injury.

What Is New Here

- $\quad$ Injury seems to be due to radiation and not to embolization.

\section{REFERENCES}

1. Sangro B, Bilbao JI, Boan J, et al. Radioembolization using Y90-resin microspheres for patients with advanced hepatocellular carcinoma. Int J Radiat Oncol Biol Phys 2006;66:792-800.

2. Lim L, Gibbs P, Yip D, et al. A prospective evaluation of treatment with Selective Internal Radiation Therapy (SIR-spheres) in patients with unresectable liver metastases from colorectal cancer previously treated with 5-FU based chemotherapy. BMC Cancer 2005;5:132.

3. Roswit B, Malsky SJ, Reid CB. Severe radiation injuries ofthe stomach, small intestine, colon and rectum. Am J Roentgenol Radium Ther Nucl Med 1972;114:460-75.

4. Coia LR, Myerson RJ, Tepper JE. Late effects of radiation therapy on the gastrointestinal tract. Int J Radiat Oncol Biol Phys 1995;31:1213-36.

5. Cosset JM, Henry-Amar M, Burgers JM, et al. Late radiation injuries of the gastrointestinal tract in the $\mathrm{H} 2$ and H5 EORTC Hodgkin's disease trials: Emphasis on the role of exploratory laparotomy and fractionation. Radiother Oncol 1988;13:61-8.

6. Breiter N, Trott KR, Sassy T. Effect of X-irradiation on the stomach of the rat. Int J Radiat Oncol Biol Phys 1989;17:779- 84.

7. Sell A, Jensen TS. Acute gastric ulcers induced by radiation. Acta Radiol Ther Phys Biol 1966;4:289-97.

8. Cosin O, Bilbao JI, Alvarez S, et al. Right gastric artery embolization prior to treatment with yttrium-90 microspheres. Cardiovasc Intervent Radiol 2007;30:98-103. 
9. Garcia C, Vicente J, Feliu J. Quimioterapia regional. In: Gonzalez Baron M, Ordóñez A, García ML, et al., eds. Oncología clínica. Fundamentos y patología general. Madrid Interamerícana: 1992:314-32.

\section{CONFLICT OF INTEREST}

The authors have declared no potential conflicts of interest. 


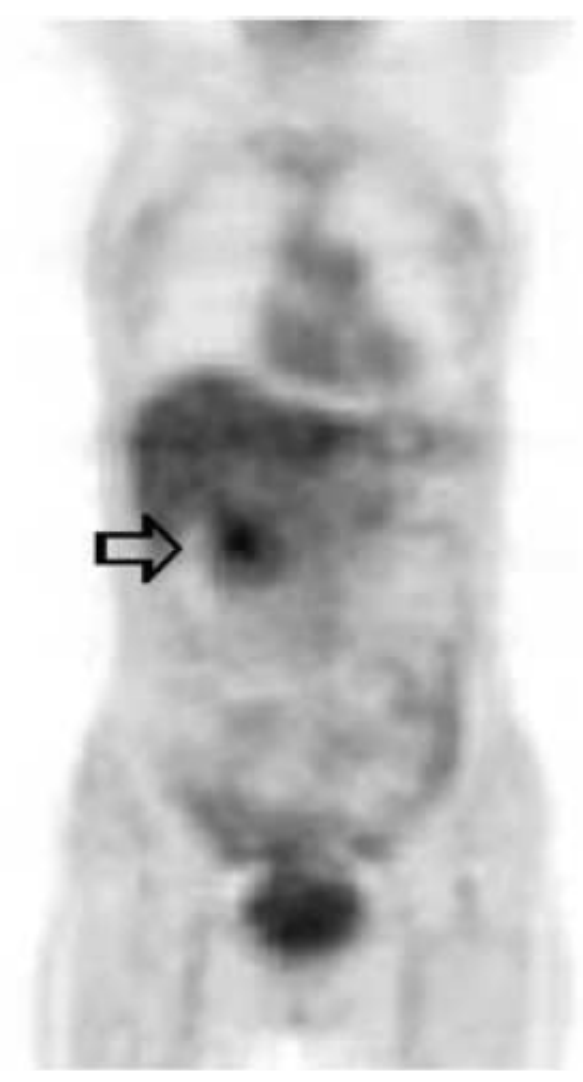

Figure 1. Hypermetabolic activity located in the pyloris of the first patient.

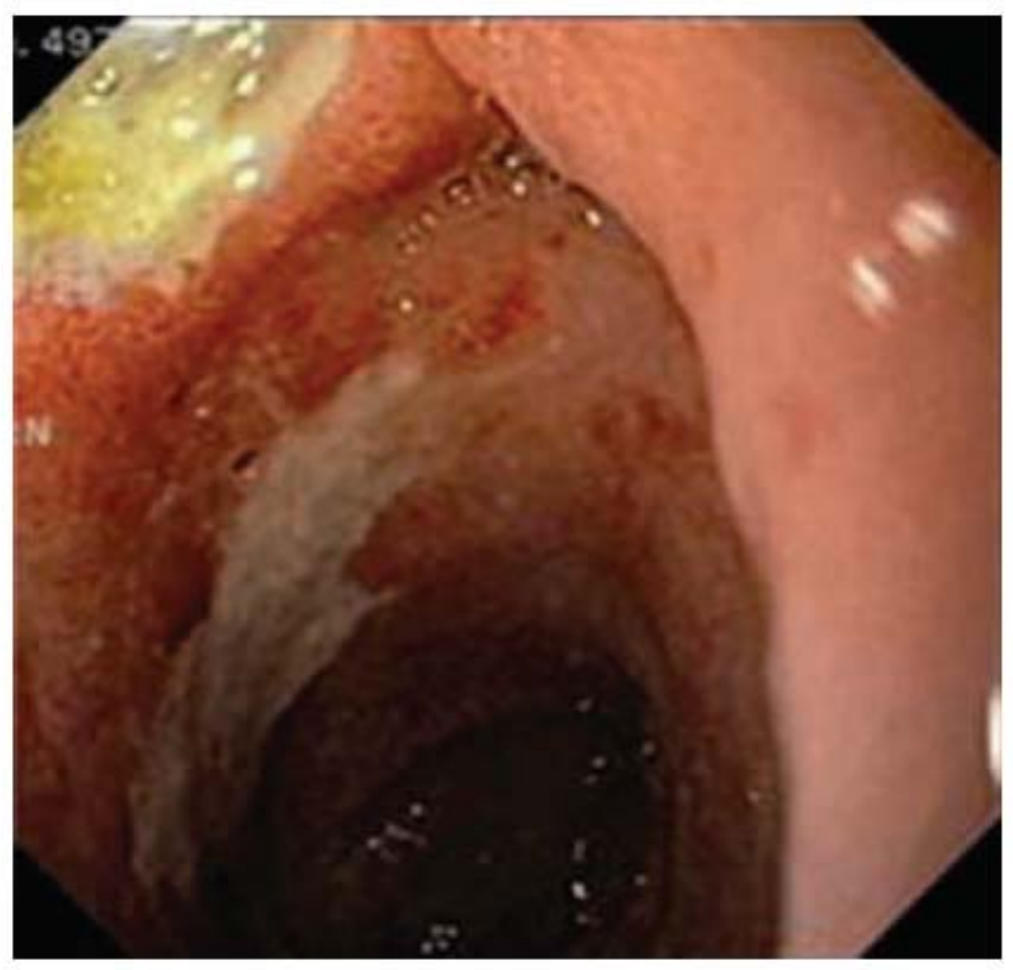

Figure 2. Duodenal ulcers after radioembolization of the first patient. 


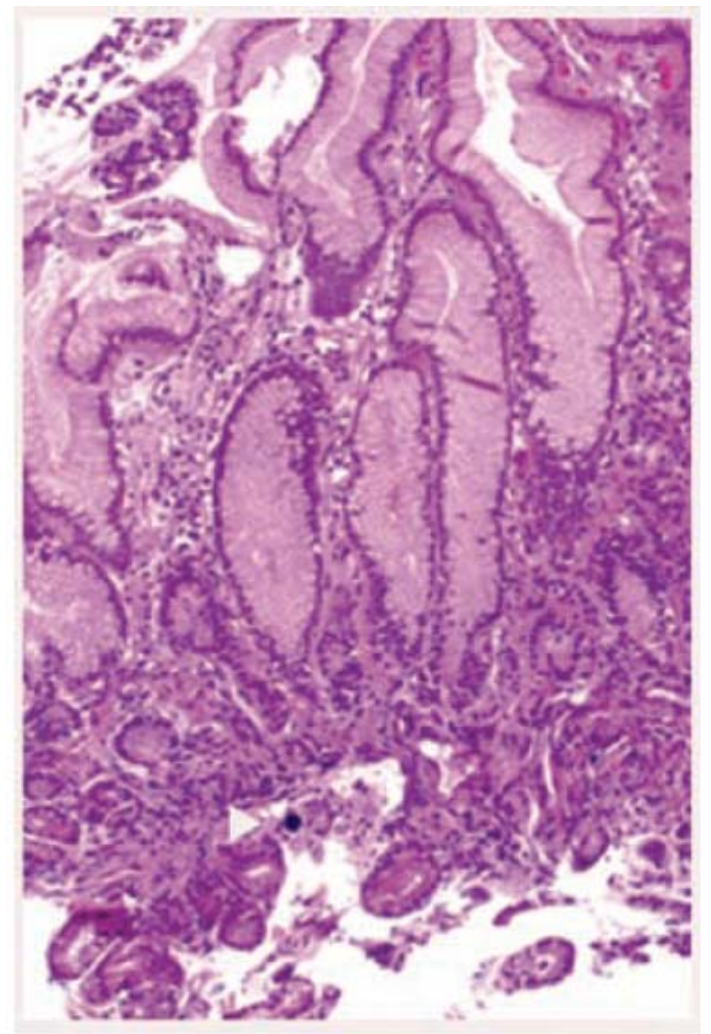

Figure 3. Small sphere in the submucosa (white arrowhead) in the biopsy of the first patient.



Figure 4. Superficial ulcer found after $1 \mathrm{yr}$ with esomeprazole and pentoxifylline (first patient). 


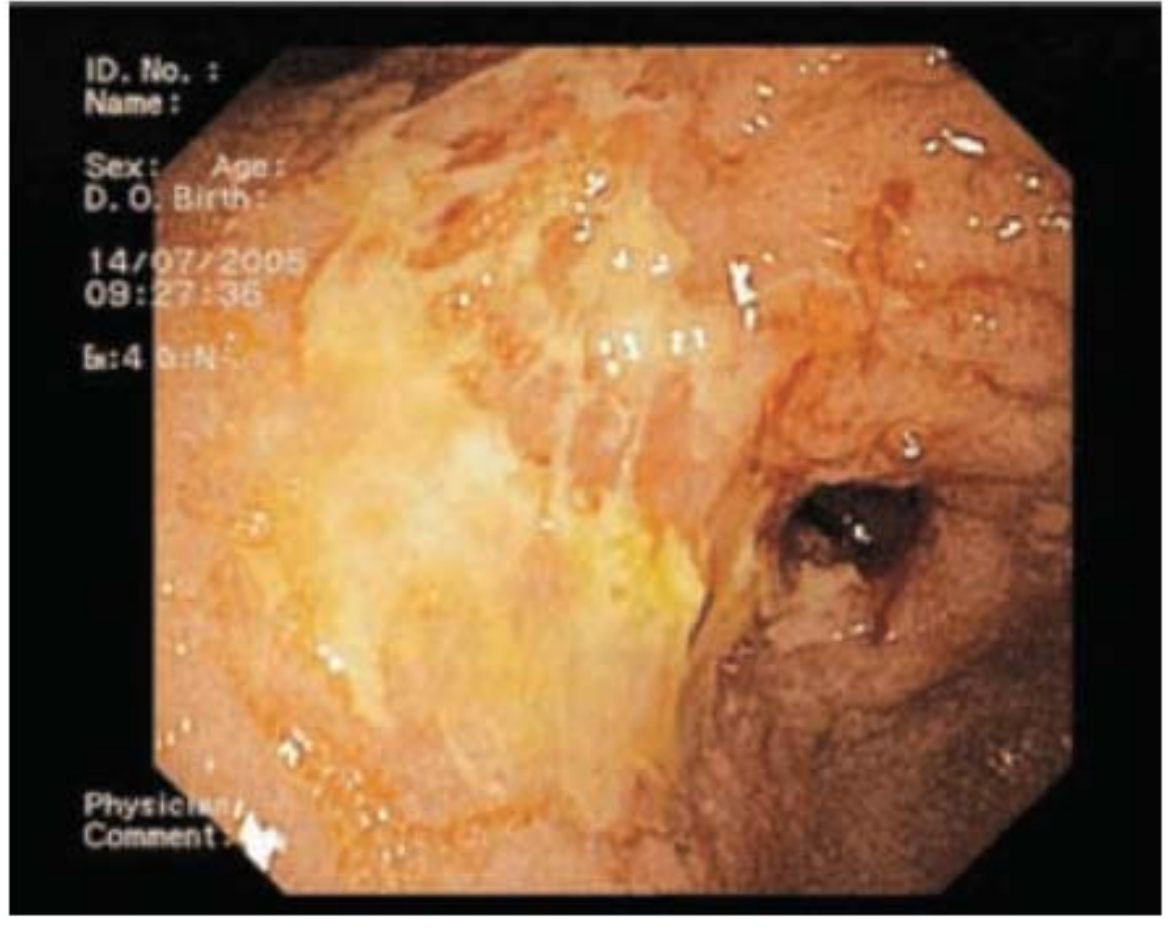

Figure 5. Ulcer along the lesser curvature in the second patient.

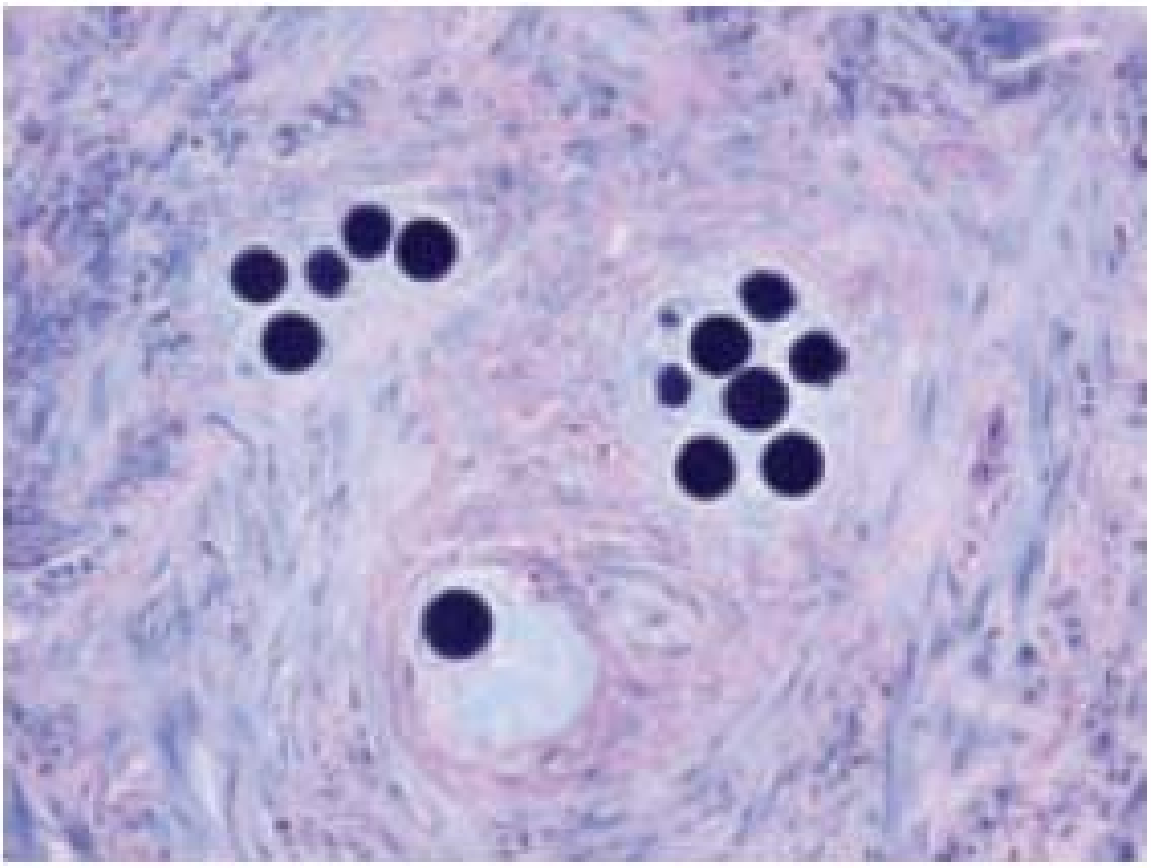

Figure 6. SIR-Spheres located inside the vessels, biopsy of the second patient. 




Figure 7. Loss of gastric mucosal outface in the third patient.

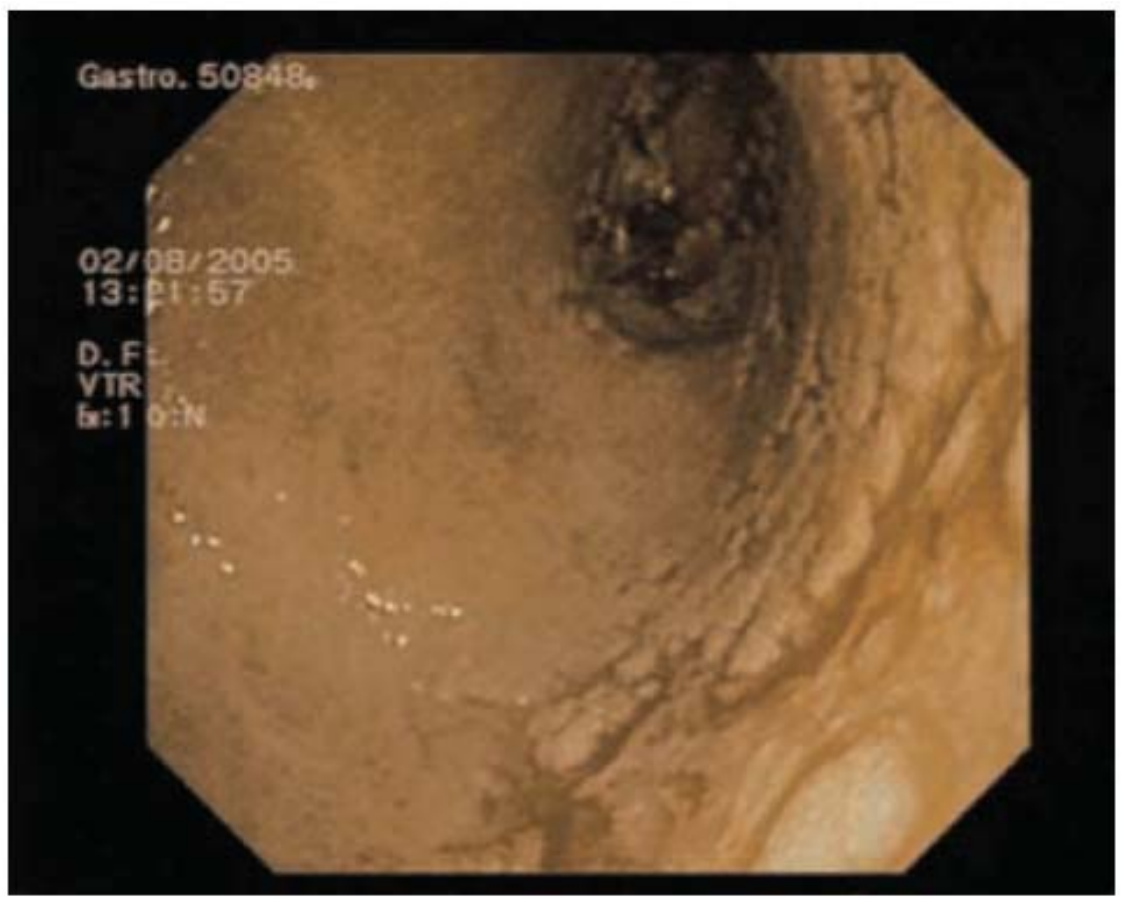

Figure 8. Denudated area with small islets of normal mucosa looking as if the mucosa was trying to regenerate. 


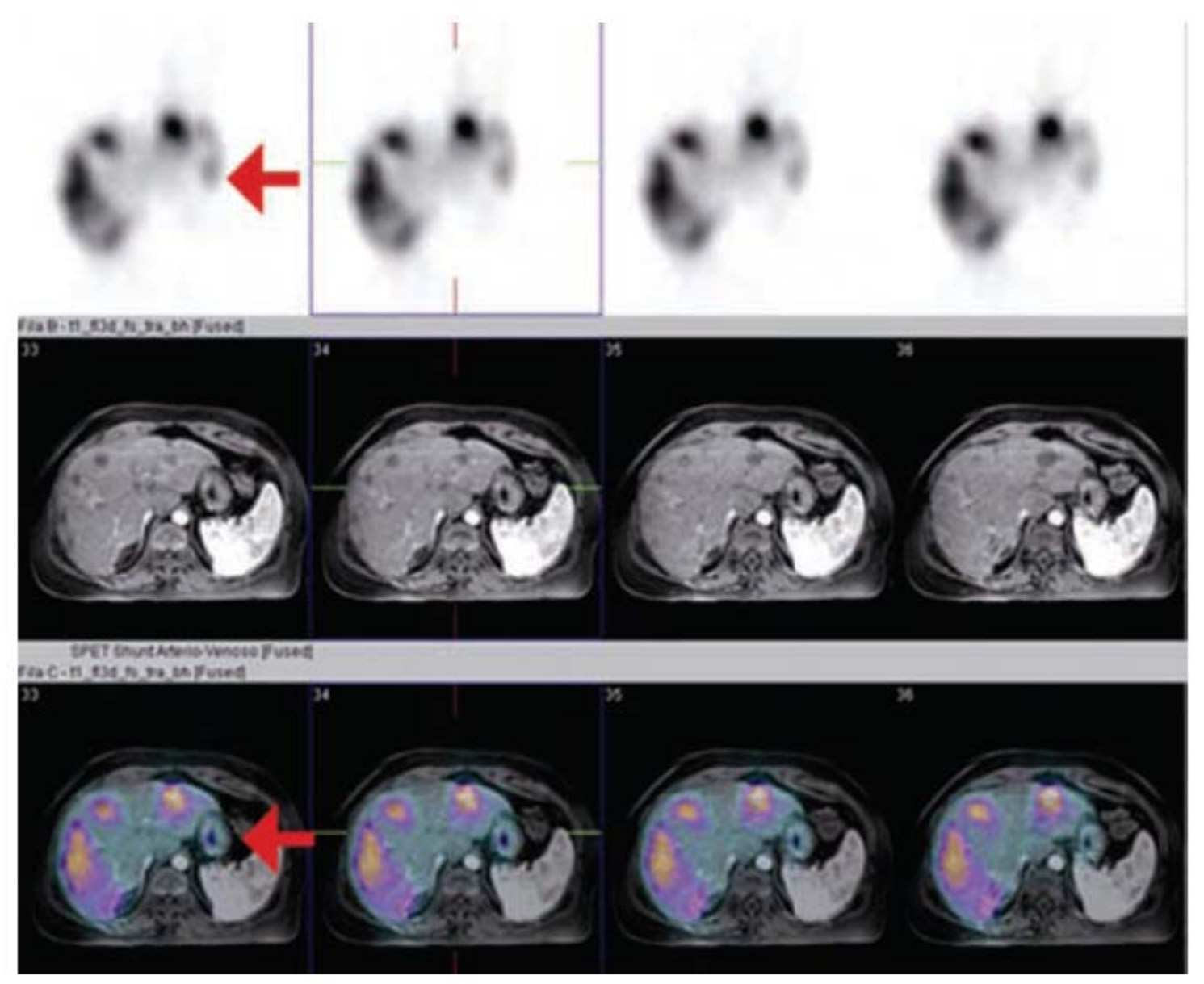

Figure 9. Fusion image (SPECT-MRI) showing no gastroduodenal activity.

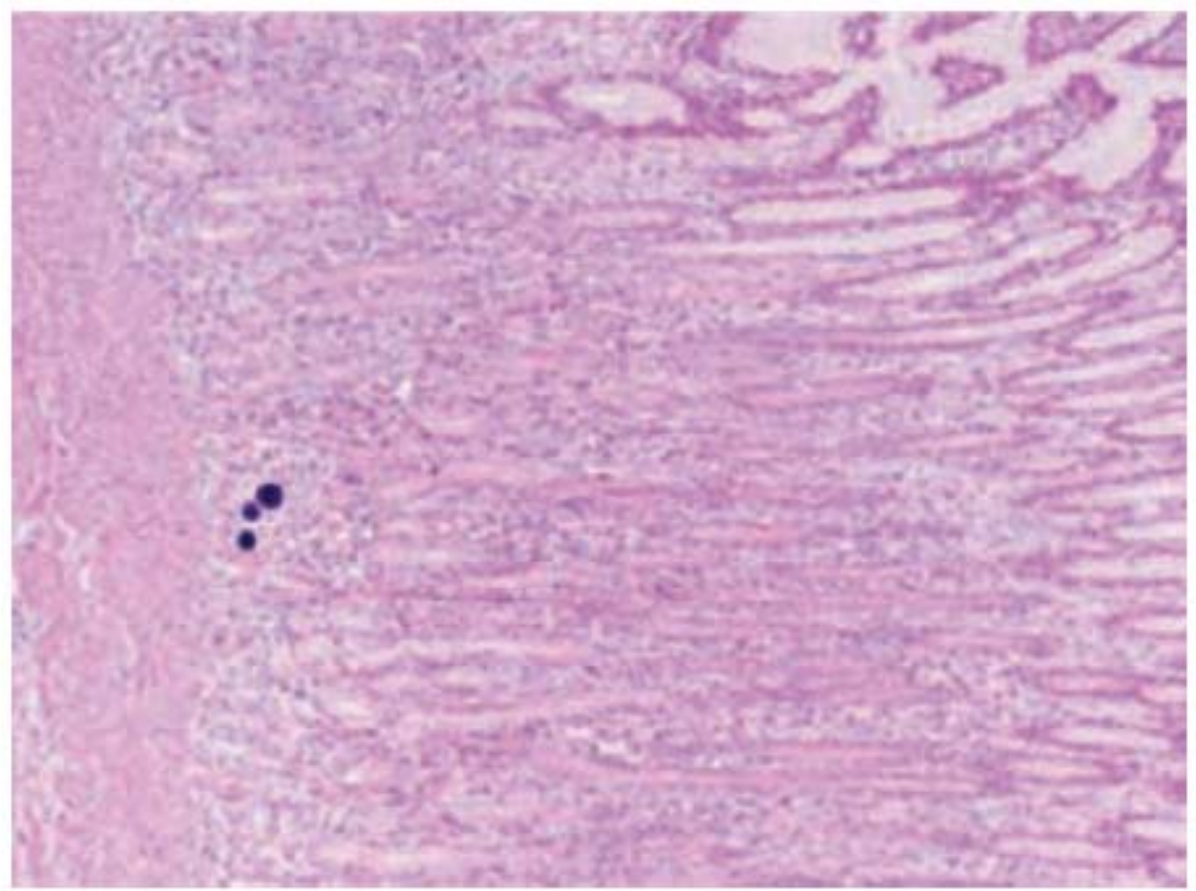

Figure 10. Cold spheres in the submucosa (animal model). 